\title{
Corrected higher order Laplacian for enhancement of pressure calculation by projection-based particle methods with applications in ocean engineering
}

\author{
Hiroyuki Ikari $^{1} \cdot$ Abbas Khayyer $^{1} \cdot$ Hitoshi Gotoh $^{1}$
}

Received: 26 September 2014 / Accepted: 30 April 2015 / Published online: 20 May 2015

(C) Springer International Publishing AG 2015

\begin{abstract}
A corrected higher order Laplacian (CHL) scheme is proposed for enhancement of pressure calculation in projection-based particle methods. The CHL scheme is derived by meticulously taking divergence of a corrected SPH gradient model in a similar manner to derivation of higher order Laplacian (HL) scheme performed by Khayyer and Gotoh (Appl Ocean Res 32(1):124-131, 2010; Appl Ocean Res 37:120-126, 2012). Unlike the original SPH gradient model considered in derivation of HL, the (firstorder) consistency of the corrected SPH gradient model is strictly guaranteed. The enhanced performance of CHL with respect to HL is shown by a set of numerical simulations corresponding to designed sinusoidal pressure oscillations, unperturbed/perturbed water jets impinging on a flat plate and a 2D diffusion problem. Hence, the CHL scheme is suggested to be applied in place of the HL one, especially for practical engineering applications including those encountered in ocean engineering.
\end{abstract}

Keywords Corrected higher order Laplacian .

Particle method - Moving particle semi-implicit method .

Pressure calculation · Consistency

\section{Introduction}

Particle methods or Lagrangian gridless methods have been increasingly applied in a wide range of engineering fields including ocean and marine engineering. In particular, successful simulations of violent sloshing flows (e.g., Gotoh

Hiroyuki Ikari

ikari@particle.kuciv.kyoto-u.ac.jp

1 Department of Civil and Earth Resources Engineering, Kyoto University, Kyoto, Japan et al. 2014; Hwang et al. 2014; Delorme et al. 2009), slamming loads on ships (e.g., Veen and Gourlay 2012) and scouring of offshore structures (e.g., Ulrich et al. 2013) have been carried out by two well-known particle methods, namely, moving particle semi-implicit (MPS; Koshizuka and Oka 1996) and smoothed particle hydrodynamics (SPH; Gingold and Monaghan 1977) methods.

Despite their robustness and wide potential range of applicability, particle methods have been suffering from major shortcomings, that is, presence of unphysical pressure oscillations that results from local particle-based interpolations by incomplete/inconsistent differential operator models (Gotoh 2009; Gotoh et al. 2013). As a result of this shortcoming, particle methods have not been extensively applied for practical ocean and marine engineering applications, particularly those corresponding to pressure calculations (e.g., wave impact pressure). Considerable efforts, however, have been made to minimize such unphysical oscillations and enhance the accuracy of particle methods by deriving corrected (e.g., Bonet and Lok 1999; Khayyer et al. 2008), higher order (e.g., Colagrossi and Landrini 2003; Khayyer and Gotoh 2009a, b) differential operator models, error mitigating terms (e.g., $\mathrm{Hu}$ and Adams 2009; Khayyer and Gotoh 2011, 2013; Kondo and Koshizuka 2011), dynamic stabilizers (e.g., Tsuruta et al. 2013), particle shifting techniques (e.g., Lind et al. 2012) and enhanced boundary conditions (e.g., Adami et al. 2012; Tsuruta et al. 2015). In the context of explicit SPH methods, a so-called delta-SPH scheme (Antuono et al. 2010, 2012) has proven to substantially enhance the pressure calculation. In a comprehensive and rigorous work, Touzé et al. (2013) highlighted the significance of higher order interpolation schemes to improve the pressure field.

In an attempt to improve the pressure calculation by a projection-based particle method, namely, MPS method, Khayyer and Gotoh (2010) derived a higher order Lapla- 
cian model (abbreviated as HL) by meticulously taking the divergence of a commonly applied SPH gradient model (Monaghan 1992) for discretization of Laplacian of pressure in the Poisson pressure equation (PPE). This derivation was later extended to 3D with verified enhancing effects (Khayyer and Gotoh 2012). One numerical issue of the HL scheme corresponds to its derivation on the basis of a SPH gradient model without a guaranteed consistency for irregularly distributed particles and/or particles without a full compact support (e.g., at and in the vicinity of free surface) (Randles and Libersky 1996; Gotoh et al. 2013; Souto-Iglesias et al. 2013). A common approach to guarantee the consistency of gradient models in particle methods is to derive corrective matrices based on Taylor-series expansions of the considered physical field (e.g., Oger et al. 2007; Khayyer and Gotoh 2011).

In this paper, a corrected higher order Laplacian, hereafter abbreviated as CHL, is derived by considering a corrected SPH gradient model and by performing a careful and meticulous derivation similar to those performed by Khayyer and Gotoh (2010, 2012). The enhanced performance of the CHL scheme will be verified by a set of simulations comprising of designed sinusoidal and exponentially excited sinusoidal pressure variations (Khayyer and Gotoh 2012), unperturbed/perturbed jets impinging on a flat plate (Molteni and Colagrossi 2009) and a 2D diffusion problem (Young et al. 2005).

\section{MPS-HS-HL-ECS-GC method}

The MPS method is a macroscopic, deterministic and projection-based particle method, initially proposed for simulation of incompressible fluid flows by Koshizuka and Oka (1996). The method reproduces the flow field by solving the continuity and Navier-Stokes equations as the governing equations. Through the past years, refined numerical schemes have been proposed in order to enhance the stability and performance of MPS method. In this study, enhanced MPS methods benefitting from so-called HS, HL (or CHL), ECS and GC schemes are considered. In this section, concise descriptions of HS, HL, ECS and GC schemes are presented in precedent order. Detailed descriptions can be found in Khayyer and Gotoh (2009a, b, 2010, 2011) and Gotoh et al. (2013).

\subsection{The HS scheme}

The HS scheme corresponds to a Higher order Source term for the PPE, incorporated for enhancement of pressure calculation. The higher order source term has been derived by considering the definition of particle number density $n$ : $n=\sum_{i \neq j} w\left(\left|\boldsymbol{r}_{j}-\boldsymbol{r}_{i}\right|\right)$

and by applying a higher order accurate time differentiation:

$$
\begin{aligned}
\frac{\mathrm{D} n}{\mathrm{D} t} & =\sum_{i \neq j} \frac{\mathrm{D} w_{i j}}{\mathrm{D} t}=\sum_{i \neq j}\left(\frac{\partial w_{i j}}{\partial r_{i j}} \frac{\partial r_{i j}}{\partial x_{i j}} \frac{\mathrm{d} x_{i j}}{\mathrm{~d} t}+\frac{\partial w_{i j}}{\partial r_{i j}} \frac{\partial r_{i j}}{\partial y_{i j}} \frac{\mathrm{d} y_{i j}}{\mathrm{~d} t}\right) \\
& =\sum_{i \neq j}\left(\frac{\partial w_{i j}}{\partial r_{i j}} \frac{x_{i j}}{r_{i j}} u_{i j}+\frac{\partial w_{i j}}{\partial r_{i j}} \frac{y_{i j}}{r_{i j}} v_{i j}\right)
\end{aligned}
$$

where $w$ symbolizes the considered kernel function, $t$ denotes time, $\boldsymbol{r}$ represents the position vector $[\boldsymbol{r}=(x, y)], u, v$ represent the components of velocity field in $x$ and $y$ directions, subscripts $i$ and $j$ correspond to a target particle $i$ and its neighboring particle $j, x_{i j}=x_{j}-x_{i}$ and $u_{i j}=u_{j}-u_{i}$. Hence, the PPE with a higher order source term would be obtained as (Khayyer and Gotoh 2009a, b):

$$
\begin{aligned}
\left(\nabla^{2} p_{k+1}\right)_{i} & =\frac{\rho}{n_{0} \Delta t}\left(\frac{\mathrm{D} n}{\mathrm{D} t}\right)^{*} \\
& =\frac{\rho}{n_{0} \Delta t} \sum_{i \neq j}\left(\frac{\partial w_{i j}}{\partial r_{i j}} \frac{x_{i j}}{r_{i j}} u_{i j}+\frac{\partial w_{i j}}{\partial r_{i j}} \frac{y_{i j}}{r_{i j}} v_{i j}\right)^{*}
\end{aligned}
$$

where $p, \rho, n_{0}$ and $\Delta t$ represent pressure, density, constant particle number density and calculation time step, respectively. The superscript $*$ denotes the pseudo time step $k+1 / 2$ (Khayyer and Gotoh 2011) with $k$ being the step of calculation.

\subsection{The HL scheme}

A key issue for improvement of pressure calculation by a projection-based particle method is to apply an accurate Laplacian model for discretization of Laplacian of pressure in the PPE [left hand side of Eq. (3)]. Khayyer and Gotoh (2010) derived a so-called higher order Laplacian model for the MPS method by meticulously taking the divergence of a gradient model. The HL scheme was founded on the following formulation:

$$
\begin{aligned}
\nabla \cdot\langle\nabla \phi\rangle_{i} & =\frac{1}{\sum_{i \neq j} w_{i j}} \sum_{i \neq j}\left(\nabla \phi_{i j} \cdot \nabla w_{i j}+\phi_{i j} \nabla^{2} w_{i j}\right) \\
& =\frac{1}{n_{0}} \sum_{i \neq j}\left(\nabla \phi_{i j} \cdot \nabla w_{i j}+\phi_{i j} \nabla^{2} w_{i j}\right)
\end{aligned}
$$

where $\langle\nabla \phi\rangle_{i}$ denotes an approximated value for gradient of an arbitrary physical quantity $\phi$ at target particle $i$. In derivation of HL scheme a commonly applied SPH gradient model (Monaghan 1992) was considered as follows: 
$\langle\nabla \phi\rangle_{i}=\frac{1}{n_{0}} \sum_{i \neq j}\left(\phi_{j}-\phi_{i}\right) \nabla w_{i j}=\frac{1}{n_{0}} \sum_{i \neq j} \phi_{i j} \nabla w_{i j}$

By considering the following definitions in 2D Cartesian coordinates

$\nabla \phi_{i j}=\frac{\partial \phi_{i j}}{\partial r_{i j}} \frac{\partial r_{i j}}{\partial x_{i j}} \boldsymbol{i}+\frac{\partial \phi_{i j}}{\partial r_{i j}} \frac{\partial r_{i j}}{\partial y_{i j}} \boldsymbol{j} ;$

$\nabla w_{i j}=\frac{\partial w_{i j}}{\partial r_{i j}} \frac{\partial r_{i j}}{\partial x_{i j}} \boldsymbol{i}+\frac{\partial w_{i j}}{\partial r_{i j}} \frac{\partial r_{i j}}{\partial y_{i j}} \boldsymbol{j}$

and performing the following derivations (Khayyer and Gotoh 2010),

$\nabla \phi_{i j} \cdot \nabla w_{i j}=\frac{\partial \phi_{i j}}{\partial r_{i j}} \frac{\partial w_{i j}}{\partial r_{i j}} \approx \frac{\phi_{j i}-\phi_{i j}}{r_{i j}} \frac{\partial w_{i j}}{\partial r_{i j}}=\frac{2 \phi_{j i}}{r_{i j}} \frac{\partial w_{i j}}{\partial r_{i j}}$

$\nabla^{2} w_{i j}=\nabla \cdot \nabla w_{i j}=\frac{\partial^{2} w_{i j}}{\partial r_{i j}^{2}}+\frac{1}{r_{i j}} \frac{\partial w_{i j}}{\partial r_{i j}}$

the HL scheme in 2D was derived as follows:

$\nabla \cdot\langle\nabla \phi\rangle_{i}=\frac{1}{n_{0}} \sum_{i \neq j}\left(\phi_{i j} \frac{\partial^{2} w_{i j}}{\partial r_{i j}^{2}}-\frac{\phi_{i j}}{r_{i j}} \frac{\partial w_{i j}}{\partial r_{i j}}\right)$

The HL scheme was shown to outperform the standard Laplacian model of MPS method which was derived on the basis of diffusion concept (Khayyer and Gotoh 2010, 2012). Except for one finite difference-based approximation, namely, $\partial \phi_{i j} / \partial r_{i j} \approx\left(\phi_{j i}-\phi_{i j}\right) / r_{i j}$, and a reasonable assumption that $\sum w_{i j}$ remains to be equal to $n_{0}$ in an incompressible fluid flow simulation, exact expressions for other terms appearing in derivation of the HL scheme were adopted. However, one clear issue related to the HL scheme is related to incompleteness or inconsistency (Liu et al. 1993; Randles and Libersky 1996) of the considered gradient model [Eq. (5)]. Thus, it is conjectured that the HL scheme will result in inaccuracies in the vicinity of boundaries with lack of a full compact support or in presence of highly-disordered particle distributions. The errors related to lack of a full compact support corresponding to discretization of PPE's Laplacian of pressure do not appear to be dominant in free-surface fluid flow simulations, as due to the dynamic free-surface boundary condition, the source term of PPE takes a zero value. Nevertheless, these errors may become considerable if the HL scheme is applied for approximation of Laplacian of another field (e.g., color function in single-phase surface tension calculations). Therefore, it is preferred to derive a so-called corrected HL (CHL) scheme with consideration of completeness (consistency) issue.

\subsection{The ECS scheme}

In order to enhance the accuracy of numerical solutions, i.e., to obtain instantaneous divergence free velocity fields, in a calculation by a projection-based particle method, Khayyer and Gotoh (2011) proposed so-called Error-Compensating terms in the Source term of PPE (abbreviated as ECS), similar to those suggested by Hu and Adams (2009) and Kondo and Koshizuka (2011). The PPE including the ECS scheme was formulated as:

$$
\begin{aligned}
\frac{\Delta t}{\rho}\left(\nabla^{2} p_{k+1}\right)_{i}= & \frac{1}{n_{0}}\left(\frac{\mathrm{D} n}{\mathrm{D} t}\right)_{i}^{*}+\mathrm{ECS}=\frac{1}{n_{0}}\left(\frac{\mathrm{D} n}{\mathrm{D} t}\right)_{i}^{*} \\
& +\alpha\left[\frac{1}{n_{0}}\left(\frac{\mathrm{D} n}{\mathrm{D} t}\right)_{i}^{k}\right]+\beta\left[\frac{1}{\Delta t} \frac{n^{k}-n_{0}}{n_{0}}\right]
\end{aligned}
$$

with dynamic coefficients $\alpha$ and $\beta$ being defined as:

$\alpha=\left|\left(\frac{n^{k}-n_{0}}{n_{0}}\right)\right| ; \quad \beta=\left|\left(\frac{\Delta t}{n_{0}}\left(\frac{\mathrm{D} n}{\mathrm{D} t}\right)_{i}^{k}\right)\right|$

\subsection{The GC scheme}

In order to obtain more accurate motions of fluid particles and minimize the probable numerical perturbations in particles' motions, a pressure Gradient Correction was proposed for the MPS method by Khayyer and Gotoh (2011). This proposal was achieved by focusing on the Taylor series expansion of pressure at a neighboring particle $j$ with respect to the pressure at a target particle $i$ (as it had been previously shown by Chen et al. 1999; Oger et al. 2007). Accordingly, a corrected pressure gradient for the MPS method was obtained as follows:

$$
\langle\nabla p\rangle_{i}=\frac{1}{n_{0}} \sum_{j \neq i} \frac{p_{j}-p_{i}}{\left|\boldsymbol{r}_{j}-\boldsymbol{r}_{i}\right|^{2}} \boldsymbol{B}_{i}\left(\boldsymbol{r}_{j}-\boldsymbol{r}_{i}\right) w\left(\left|\boldsymbol{r}_{j}-\boldsymbol{r}_{i}\right|\right)
$$

with the corrective matrix $\boldsymbol{B}$ being formulated as:

$$
\boldsymbol{B}_{i}=\left(\begin{array}{ll}
\sum V_{i j} \frac{w_{i j} x_{i j}^{2}}{r_{i j}^{2}} & \sum V_{i j} \frac{w_{i j} x_{i j} y_{i j}}{r_{i j}^{2}} \\
\sum V_{i j} \frac{w_{i j} x_{i j} y_{i j}}{r_{i j}^{2}} \sum V_{i j} \frac{w_{i j} y_{i j}^{2}}{r_{i j}^{2}}
\end{array}\right)^{-1} ; \quad V_{i j}=\frac{1}{n_{0}}
$$

The HS, ECS and GC schemes are applied in all the simulations performed in this paper.

\section{Corrected higher order Laplacian (CHL) scheme}

As it was previously discussed in Sect. 2.2, a clear shortcoming of HL scheme is related to incompleteness of the gradient 
model considered for derivation of this scheme. The basis of gradient correction corresponds to a Taylor-series expansion of an arbitrary physical field $\phi$ at a neighboring particle $j$ based on information at a target particle $i$ as follows:

$\phi_{j}=\phi_{i}+\left(\frac{\partial \phi}{\partial x}\right)_{i} x_{i j}+\left(\frac{\partial \phi}{\partial y}\right)_{i} y_{i j} ; \quad x_{i j}=x_{j}-x_{i}$

Multiplying both hand sides by $\int \frac{\partial w}{\partial r} \frac{\boldsymbol{r}_{i j}}{\left|\boldsymbol{r}_{i j}\right|} \mathrm{d} \boldsymbol{r}$, yields:

$$
\begin{aligned}
& \int\left(\phi_{j}-\phi_{i}\right)\left(\frac{\partial w}{\partial r} \frac{x_{i j}}{\left|\boldsymbol{r}_{i j}\right|} \mathrm{d} x+\frac{\partial w}{\partial r} \frac{y_{i j}}{\left|\boldsymbol{r}_{i j}\right|} \mathrm{d} y\right) \\
& =\left(\frac{\partial \phi}{\partial x}\right)_{i} \int\left(x_{i j} \frac{\partial w}{\partial r} \frac{x_{i j}}{\left|\boldsymbol{r}_{i j}\right|} \mathrm{d} x+x_{i j} \frac{\partial w}{\partial r} \frac{y_{i j}}{\left|\boldsymbol{r}_{i j}\right|} \mathrm{d} y\right) \\
& +\left(\frac{\partial \phi}{\partial y}\right)_{i} \int\left(y_{i j} \frac{\partial w}{\partial r} \frac{x_{i j}}{\left|\boldsymbol{r}_{i j}\right|} \mathrm{d} x+y_{i j} \frac{\partial w}{\partial r} \frac{y_{i j}}{\left|\boldsymbol{r}_{i j}\right|} \mathrm{d} y\right)
\end{aligned}
$$

where $\boldsymbol{r}_{i j}=\left(x_{i j}, y_{i j}\right)$. From Eq. (15) the following corrected gradient vector will be obtained (Randles and Libersky 1996):

$$
\left(\begin{array}{l}
\frac{\partial \phi}{\partial x} \\
\frac{\partial \phi}{\partial y}
\end{array}\right)_{i}=-\int\left(\phi_{j}-\phi_{i}\right) \boldsymbol{C}_{i} \frac{\partial w}{\partial r} \frac{\boldsymbol{r}_{i j}}{\left|\boldsymbol{r}_{i j}\right|} \mathrm{d} \boldsymbol{r}
$$

where $\boldsymbol{C}$ is a corrective matrix for target particle $i$, formulated as:

$$
\boldsymbol{C}_{i}=\left(\begin{array}{l}
-\int \frac{\partial w}{\partial r} \frac{x_{i j}^{2}}{\left|\boldsymbol{r}_{i j}\right|} \mathrm{d} x-\int \frac{\partial w}{\partial r} \frac{x_{i j} y_{i j}}{\left|\boldsymbol{r}_{i j}\right|} \mathrm{d} x \\
-\int \frac{\partial w}{\partial r} \frac{x_{i j} y_{i j}}{\left|\boldsymbol{r}_{i j}\right|} \mathrm{d} y-\int \frac{\partial w}{\partial r} \frac{y_{i j}^{2}}{\left|\boldsymbol{r}_{i j}\right|} \mathrm{d} y
\end{array}\right)^{-1}
$$

The above corrective matrix ensures the first-order consistency of approximations (e.g., Oger et al. 2007; Gotoh et al. 2013). In discrete form, the corrected gradient model and the corresponding corrective matrix are expressed by Eqs. (18) and (19).

$$
\begin{aligned}
\langle\nabla \phi\rangle_{i} & =-\frac{1}{n_{0}} \sum_{j \neq i} \phi_{i j} \boldsymbol{C}_{i} \nabla_{i j} w_{i j} \\
& =-\frac{1}{n_{0}} \sum_{j \neq i} \phi_{i j} \boldsymbol{C}_{i} \frac{\partial w}{\partial r} \frac{\boldsymbol{r}_{i j}}{\left|\boldsymbol{r}_{i j}\right|} \\
\boldsymbol{C}_{i} & =\left(\begin{array}{l}
-\frac{1}{n_{0}} \sum_{i \neq j} \frac{\partial w}{\partial r} \frac{x_{i j}^{2}}{\left|\boldsymbol{r}_{i j}\right|}-\frac{1}{n_{0}} \sum_{i \neq j} \frac{\partial w}{\partial r} \frac{x_{i j} y_{i j}}{\left|\boldsymbol{r}_{i j}\right|} \\
-\frac{1}{n_{0}} \sum_{i \neq j} \frac{\partial w}{\partial r} \frac{x_{i j} y_{i j}}{\left|\boldsymbol{r}_{i j}\right|}-\frac{1}{n_{0}} \sum_{i \neq j} \frac{\partial w}{\partial r} \frac{y_{i j}^{2}}{\left|\boldsymbol{r}_{i j}\right|}
\end{array}\right)
\end{aligned}
$$

Similar to derivation of HL scheme, the Laplacian of $\phi$ is considered to be obtained by taking the divergence of gradient of $\phi$. Hence,

$$
\begin{aligned}
\left\langle\nabla^{2} \phi\right\rangle_{i} & =\nabla_{i} \cdot\langle\nabla \phi\rangle_{i} \\
& =\frac{1}{n_{0}} \sum_{j \neq i}\left\{\nabla_{i j} \phi_{i j} \cdot \boldsymbol{C}_{i} \nabla_{i j} w_{i j}+\phi_{i j} \nabla_{i j} \cdot\left(\boldsymbol{C}_{i} \nabla_{i j} w_{i j}\right)\right\}
\end{aligned}
$$

where

$\nabla_{i}=\left(\frac{\partial}{\partial x_{i}} \frac{\partial}{\partial y_{i}}\right), \quad \nabla_{i j}=\left(\frac{\partial}{\partial x_{i j}} \frac{\partial}{\partial y_{i j}}\right)$

and thus,

$\nabla_{i}=\left(\frac{\partial}{\partial x_{i j}} \frac{\partial x_{i j}}{\partial x_{i}} \frac{\partial}{\partial y_{i j}} \frac{\partial y_{i j}}{\partial y_{i}}\right)=-\nabla_{i j}$

Accordingly the first term on the right hand side of Eq. (20) will be expressed as:

$$
\begin{aligned}
\nabla \phi_{i j} \cdot \boldsymbol{C}_{i} \nabla_{i j} w_{i j} \\
=\frac{\partial \phi_{i j}}{\partial r_{i j}} \frac{\partial r_{i j}}{\partial x_{i j}}\left(C_{11} \frac{\partial w_{i j}}{\partial r_{i j}} \frac{\partial r_{i j}}{\partial x_{i j}}+C_{12} \frac{\partial w_{i j}}{\partial r_{i j}} \frac{\partial r_{i j}}{\partial y_{i j}}\right) \\
+\frac{\partial \phi_{i j}}{\partial r_{i j}} \frac{\partial r_{i j}}{\partial y_{i j}}\left(C_{21} \frac{\partial w_{i j}}{\partial r_{i j}} \frac{\partial r_{i j}}{\partial x_{i j}}+C_{22} \frac{\partial w_{i j}}{\partial r_{i j}} \frac{\partial r_{i j}}{\partial y_{i j}}\right) \\
=\frac{\phi_{j i}-\phi_{i j}}{\left|\boldsymbol{r}_{i j}\right|} \frac{x_{i j}}{\left|\boldsymbol{r}_{i j}\right|}\left(C_{11} \frac{\partial w_{i j}}{\partial r_{i j}} \frac{x_{i j}}{\left|\boldsymbol{r}_{i j}\right|}+C_{12} \frac{\partial w_{i j}}{\partial r_{i j}} \frac{y_{i j}}{\left|\boldsymbol{r}_{i j}\right|}\right) \\
+\frac{\phi_{j i}-\phi_{i j}}{\left|\boldsymbol{r}_{i j}\right|} \frac{y_{i j}}{\left|\boldsymbol{r}_{i j}\right|}\left(C_{21} \frac{\partial w_{i j}}{\partial r_{i j}} \frac{x_{i j}}{\left|\boldsymbol{r}_{i j}\right|}+C_{22} \frac{\partial w_{i j}}{\partial r_{i j}} \frac{y_{i j}}{\left|\boldsymbol{r}_{i j}\right|}\right) \\
=-\frac{2 \phi_{i j}}{\left|\boldsymbol{r}_{i j}\right|^{3}} \frac{\partial w_{i j}}{\partial r_{i j}}\left\{C_{11} x_{i j}^{2}+\left(C_{12}+C_{21}\right) x_{i j} y_{i j}+C_{22} y_{i j}^{2}\right\}
\end{aligned}
$$

And for derivation of second term, divergence of the product of $\boldsymbol{C}$ and $\nabla_{i j} w_{i j}$ will be expressed as:

$$
\begin{aligned}
\nabla_{i j} \cdot\left(\boldsymbol{C}_{i} \nabla_{i j} w_{i j}\right)= & \frac{\partial}{\partial x_{i j}}\left(C_{11} \frac{\partial w_{i j}}{\partial r_{i j}} \frac{\partial r_{i j}}{\partial x_{i j}}+C_{12} \frac{\partial w_{i j}}{\partial r_{i j}} \frac{\partial r_{i j}}{\partial y_{i j}}\right) \\
& +\frac{\partial}{\partial y_{i j}}\left(C_{21} \frac{\partial w_{i j}}{\partial r_{i j}} \frac{\partial r_{i j}}{\partial x_{i j}}+C_{22} \frac{\partial w_{i j}}{\partial r_{i j}} \frac{\partial r_{i j}}{\partial y_{i j}}\right) \\
= & \frac{\partial C_{11}}{\partial x_{i j}} \frac{\partial w_{i j}}{\partial r_{i j}} \frac{x_{i j}}{\left|\boldsymbol{r}_{i j}\right|}+C_{11} \frac{x_{i j}^{2}}{\left|\boldsymbol{r}_{i j}\right|^{2}} \frac{\partial^{2} w_{i j}}{\partial r_{i j}^{2}} \\
& +C_{11} \frac{\partial w_{i j}}{\partial r_{i j}} \frac{1}{\left|\boldsymbol{r}_{i j}\right|}-C_{11} \frac{\partial w_{i j}}{\partial r_{i j}} \frac{x_{i j}^{2}}{\left|\boldsymbol{r}_{i j}\right|^{3}} \\
& +\frac{\partial C_{12}}{\partial x_{i j}} \frac{\partial w_{i j}}{\partial r_{i j}} \frac{y_{i j}}{\left|\boldsymbol{r}_{i j}\right|}+C_{12} \frac{x_{i j}}{\left|\boldsymbol{r}_{i j}\right|^{2}} \frac{\partial^{2} w_{i j}}{\partial r_{i j}^{2}} \\
& -C_{12} \frac{\partial w_{i j}}{\partial r_{i j}} \frac{x_{i j} y_{i j}}{\left|\boldsymbol{r}_{i j}\right|^{3}}+\frac{\partial C_{21}}{\partial y_{i j}} \frac{\partial w_{i j}}{\partial r_{i j}} \frac{x_{i j}}{\left|\boldsymbol{r}_{i j}\right|} \\
& +C_{21} \frac{x_{i j} y_{i j}}{\left|\boldsymbol{r}_{i j}\right|^{2}} \frac{\partial^{2} w_{i j}}{\partial r_{i j}^{2}}-C_{21} \frac{\partial w_{i j}}{\partial r_{i j}} \frac{x_{i j} y_{i j}}{\left|\boldsymbol{r}_{i j}\right|^{3}}
\end{aligned}
$$




$$
\begin{aligned}
& +\frac{\partial C_{22}}{\partial y_{i j}} \frac{\partial w_{i j}}{\partial r_{i j}} \frac{y_{i j}}{\left|\boldsymbol{r}_{i j}\right|}+C_{22} \frac{y_{i j}^{2}}{\left|\boldsymbol{r}_{i j}\right|^{2}} \frac{\partial^{2} w_{i j}}{\partial r_{i j}^{2}} \\
& +C_{22} \frac{\partial w_{i j}}{\partial r_{i j}} \frac{1}{\left|\boldsymbol{r}_{i j}\right|}-C_{22} \frac{\partial w_{i j}}{\partial r_{i j}} \frac{y_{i j}^{2}}{\left|\boldsymbol{r}_{i j}\right|^{3}} \\
& =\left(\frac{1}{\left|\boldsymbol{r}_{i j}\right|^{2}} \frac{\partial^{2} w_{i j}}{\partial r_{i j}^{2}}-\frac{1}{\left|\boldsymbol{r}_{i j}\right|^{3}} \frac{\partial w_{i j}}{\partial r_{i j}}\right) \\
& \times\left\{C_{11} x_{i j}^{2}+\left(C_{12}+C_{21}\right) x_{i j} y_{i j}+C_{22} y_{i j}^{2}\right\} \\
& +\frac{1}{\left|\boldsymbol{r}_{i j}\right|} \frac{\partial w_{i j}}{\partial r_{i j}}\left(C_{11}+C_{22}\right) \\
& +\frac{x_{i j}}{\left|\boldsymbol{r}_{i j}\right|} \frac{\partial w_{i j}}{\partial r_{i j}}\left(\frac{\partial C_{11}}{\partial x_{i j}}+\frac{\partial C_{21}}{\partial y_{i j}}\right) \\
& +\frac{y_{i j}}{\left|\boldsymbol{r}_{i j}\right|} \frac{\partial w_{i j}}{\partial r_{i j}}\left(\frac{\partial C_{12}}{\partial x_{i j}}+\frac{\partial C_{22}}{\partial y_{i j}}\right) \\
& =\left(\frac{1}{\left|\boldsymbol{r}_{i j}\right|^{2}} \frac{\partial^{2} w_{i j}}{\partial r_{i j}^{2}}-\frac{1}{\left|\boldsymbol{r}_{i j}\right|^{3}} \frac{\partial w_{i j}}{\partial r_{i j}}\right) \\
& \times\left\{C_{11} x_{i j}^{2}+\left(C_{12}+C_{21}\right) x_{i j} y_{i j}+C_{22} y_{i j}^{2}\right\} \\
& +\frac{1}{\left|\boldsymbol{r}_{i j}\right|} \frac{\partial w_{i j}}{\partial r_{i j}}\left(C_{11}+C_{22}\right) \\
& -\frac{x_{i j}}{\left|\boldsymbol{r}_{i j}\right|^{3}} \frac{\partial w_{i j}}{\partial r_{i j}}\left(C_{11} x_{i j}+C_{21} y_{i j}\right) \\
& -\frac{y_{i j}}{\left|\boldsymbol{r}_{i j}\right|^{3}} \frac{\partial w_{i j}}{\partial r_{i j}}\left(C_{12} x_{i j}+C_{22} y_{i j}\right)
\end{aligned}
$$

It should be noted here that,

$\frac{\partial C_{\alpha \beta}}{\partial x_{i j}}=\frac{\partial C_{\alpha \beta}}{\partial r_{i j}} \frac{\partial r_{i j}}{\partial x_{i j}} \approx \frac{0-C_{\alpha \beta}}{\left|\boldsymbol{r}_{i j}\right|} \frac{x_{i j}}{\left|\boldsymbol{r}_{i j}\right|}=-\frac{C_{\alpha \beta} x_{i j}}{\left|\boldsymbol{r}_{i j}\right|^{2}}$

The CHL scheme is thus obtained by considering Eqs. (20), (23) and (24). The kernel function applied in all simulations of this study, and for all the schemes, is a Wendland one (Wendland 1995), formulated as follows:

$w(r)= \begin{cases}\left(1-\frac{r}{r_{e}}\right)^{4}\left(1+4 \frac{r}{r_{e}}\right) & \left(0<r \leq r_{e}\right) \\ 0 & \left(r>r_{e}\right)\end{cases}$

where $r_{e}$ represents the radius of influence circle for a typical target particle. In all the performed simulations of this paper $r_{e}=2.4 d_{0}$.

\section{Verification tests}

The enhancing performance of the CHL scheme with respect to the HL scheme will be shown by simulations of designed sinusoidal and exponentially excited sinusoidal pressure variations (Khayyer and Gotoh 2012), unperturbed/perturbed jets impinging on a flat plate (Molteni and Colagrossi 2009) and a 2D diffusion problem (Young et al. 2005). The simulations are performed by an improved version of the MPS method, namely, MPS-HS-ECS-GC method (Gotoh et al. 2013; Khayyer and Gotoh 2011), briefly described in Sect. 2, incorporated with either HL or CHL schemes (MPS-HS-HLECS-GC or MPS-HS-CHL-ECS-GC).

\subsection{Designed sinusoidal pressure variations (Khayyer and Gotoh 2012)}

Designed sinusoidal pressure variations are carried out to investigate the performance of newly proposed CHL scheme with respect to the HL one. Detailed descriptions regarding this test have been provided by Khayyer and Gotoh (2011, 2012). Figure 1 shows a schematic sketch of the computational domain as well as the simulation conditions. The modified gravitational acceleration considered for this test is defined as follows:

$\boldsymbol{g}_{d}(t)=2 \boldsymbol{g}+\delta \boldsymbol{g} \sin \left(\frac{2 \pi t}{T}\right)$

where $\boldsymbol{g}_{d}$ represents designed external accelerations applied to fluid particles, $\boldsymbol{g}$ is the Earth's gravitational acceleration $\left[=(0,-9.81) \mathrm{m} / \mathrm{s}^{2}\right], t$ stands for the simulation time and $T$ denotes the period of sinusoidal term variations $(=0.01 \mathrm{~s})$. The constant coefficient $\delta$ is selected to be 0.4 . The time increment of calculation, $\Delta t$, is set according to the Courant stability condition and a (maximum allowable) time resolution chosen as $2.0 \mathrm{E}-5$, that is:

$\Delta t=\min \left(\kappa d_{0} / u_{\max }, 2.0 \mathrm{E}-5\right)$

where $\kappa=$ ratio of the time step to Courant number $(=0.2)$ and $u_{\max }=$ maximum instantaneous velocity of particles. The above time increment is considered for all the simulations performed in this section regardless of the selected particle size.

Figure 2 illustrates a qualitative comparison in between the spatial distributions of pressure obtained by applying the HL scheme and the CHL one by considering a set of three spatial resolutions (particle sizes) of (a) $d_{0}=10 \mathrm{~mm}$, (b) $d_{0}=5 \mathrm{~mm}$ and (c) $d_{0}=2.5 \mathrm{~mm}$. In all the three considered cases, application of CHL has resulted in a clear enhancement

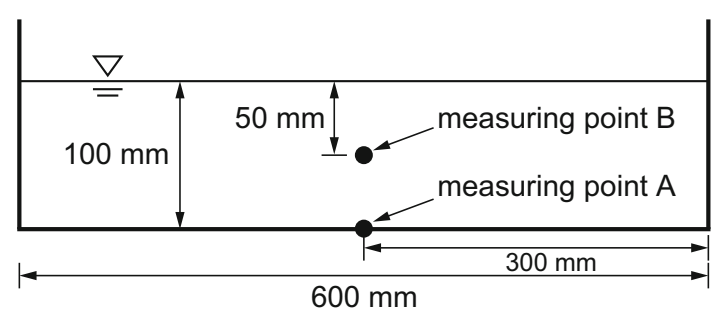

Fig. 1 Schematic sketch of the computational domain — designed sinusoidal pressure variations 
Fig. 2 Snapshots of particle together with pressure field for a set of spatial resolutions of a $d_{0}=10 \mathrm{~mm}, \mathbf{b} d_{0}=5 \mathrm{~mm}$ and $\mathbf{c} d_{0}=2.5 \mathrm{~mm}$ by HL (higher order Laplacian) and CHL (corrected higher order Laplacian) schemes-designed sinusoidal pressure variations

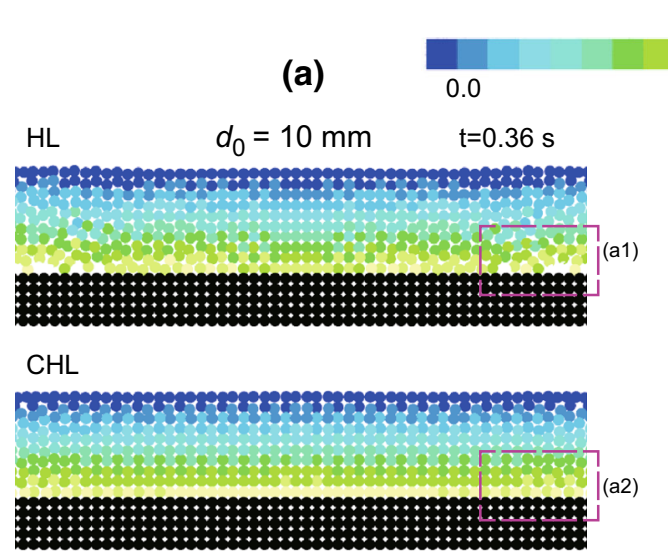

(c)

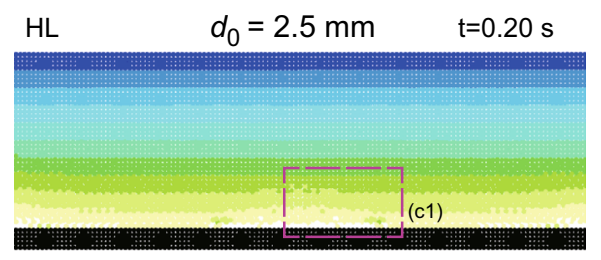

$\mathrm{CHL}$

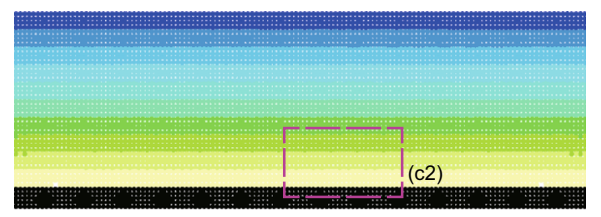

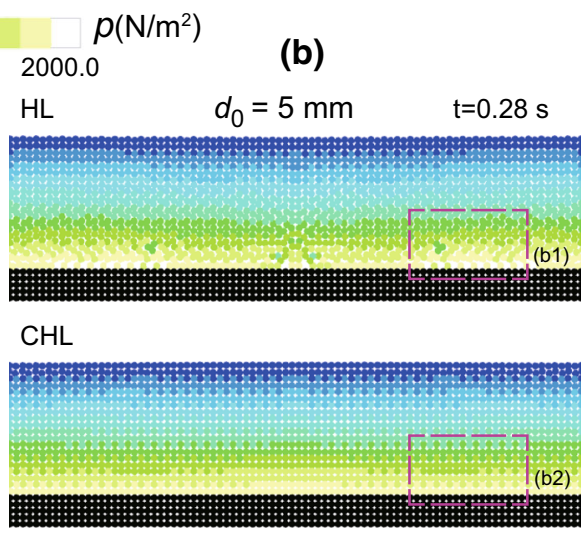

(a1)

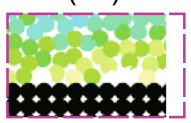

(b1)

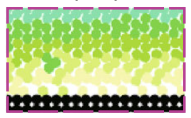

(c1) (a2)

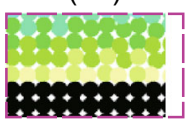

(b2)

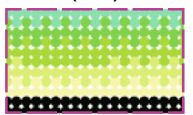

(c2)
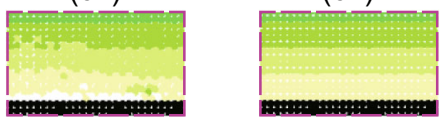

of spatial distribution of pressure providing a more regular and smoother pressure field.

Figure 3 depicts the time histories of calculated and analytical pressure at measuring point A. This figure approves the enhancing effect of CHL in a quantitative manner, where significantly enhanced pressure calculations are obtained by applying the CHL scheme in all three considered spatial resolutions. There appears to be a deterioration of simulation results for both HL and CHL by refinement of spatial resolution (particle size). However, this apparent inconsistency is likely caused by consideration of a similar maximum allowable time resolution for all the simulations. In other words, we should have set smaller maximum allowable time resolutions as the particle size was being refined, but on the other hand, we preferred to set exactly similar calculation conditions for all the performed simulations regardless of particle size, due to simplicity and clarity, as well as the main target of our present study, i.e., investigation of the performance of CHL with respect to HL. We admit that a detailed and rigorous study on the convergence properties of MPS-based methods employing either HL or CHL must be carried out.

Figure 4 portrays the effect of CHL in improvement of volume conservation which is an important aspect in particle method simulations, especially those corresponding to incompressible fluid flows. This figure shows time variations of calculated particle number density at measuring point B normalized by the initial one $\left(n_{0}\right)$. From Fig. 4 , the error in volume conservation related to HL scheme reaches more than $10 \%$, while, this error for CHL scheme tends to be confined within a limited range with maximum observed error of about $2 \%$. As illustrated by Khayyer and Gotoh (2011, 2013), volume conservation plays a crucial role both in pressure calculation and overall stability of the method.

Figure 5 shows the spatial distribution of particles together with pressure field for an initially irregular and disordered distribution of particles where initially (at $t=0$ ) all the fluid particles were randomly displaced by $0.05 d_{0}$ in both horizontal and vertical directions. The presented figure approves the enhancing effects of CHL in providing a more regular and smoother pressure field for an initially irregular particle distribution. It is worth to mention here that in a recent work, Antuono et al. (2014) proposed an algorithm to measure disorder in particle methods and highlighted the applications of their proposed disorder measure in inspecting the correlation in between the measure of disorder and accuracy of particle methods.

Figure 6 depicts the time histories of calculated pressure as well as analytical solution at measuring point $\mathrm{A}$, for the initially irregular particle distribution. From Fig. 6, application of CHL has been effective in providing a more accurate 


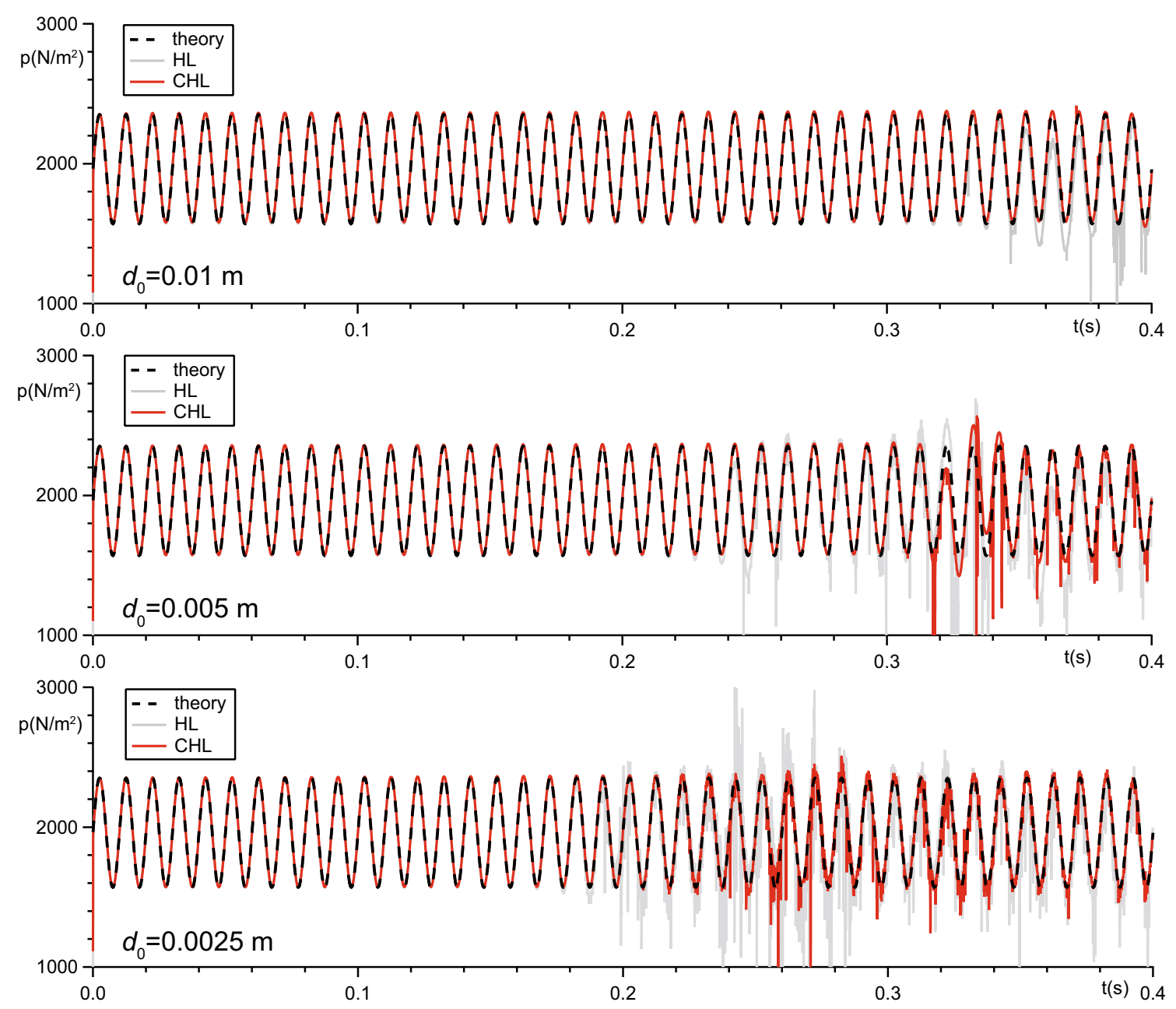

Fig. 3 Time histories of calculated and analytical pressure at measuring point A—designed sinusoidal pressure variations—regular initial particle distribution

pressure calculation with minimized unphysical oscillations in time domain.

\subsection{Designed exponentially excited sinusoidal pressure variations (Khayyer and Gotoh 2012)}

In order to further investigate the performance of CHL with respect to HL, a set of exponentially excited sinusoidal pressure variations is performed by considering three different spatial resolutions $\left(d_{0}=0.01,0.005\right.$ and $\left.0.0025 \mathrm{~m}\right)$. The computational domain corresponds to that of Sect. 4.1 schematically shown in Fig. 1. Here, the designed gravitational acceleration is considered as:

$\boldsymbol{g}_{d}(t)=2 \boldsymbol{g}+\delta \boldsymbol{g} \sin \left(\frac{2 \pi t}{T}\right) \exp \left(\frac{\varepsilon \pi t}{T}\right)$

where $T$, the period of sinusoidal term variations, is set as $0.01 \mathrm{~s}$ and the constant coefficients $\delta$ and $\varepsilon$ are selected to be 0.2 and 0.015 , respectively. In order to see the differences in between performances of CHL and HL schemes more clearly, an initially irregular distribution of particles is considered, similar to that in Sect. 4.1, i.e., all the fluid particles were randomly displaced by $0.05 d_{0}$ in both horizontal and vertical directions. The calculation time step, $\Delta t$, is set according to the Courant stability condition and a time resolution chosen as $2.0 \mathrm{E}-5$.

Figure 7 depicts the time histories of pressure variations at measuring point A (Fig. 1). As it can be clearly seen from this figure, in all the three performed simulations, the CHL scheme outperforms the HL one, in providing a more accurate calculation of pressure field. Table 1 shows the RMSE (root mean square error) corresponding to these three simulations. Although the CHL evidently outperforms the HL, a clear convergence cannot be seen. This is most probably due to the fact that the maximum allowable time resolutions for all the performed three cases were the same. A detailed further study on convergence properties of both HL and CHL schemes appears to be indispensable. 
Fig. 4 Time variations of calculated particle number density at measuring point $B$ normalized by the initial one $\left(n_{0}\right)$-designed sinusoidal pressure variations-regular initial particle distribution
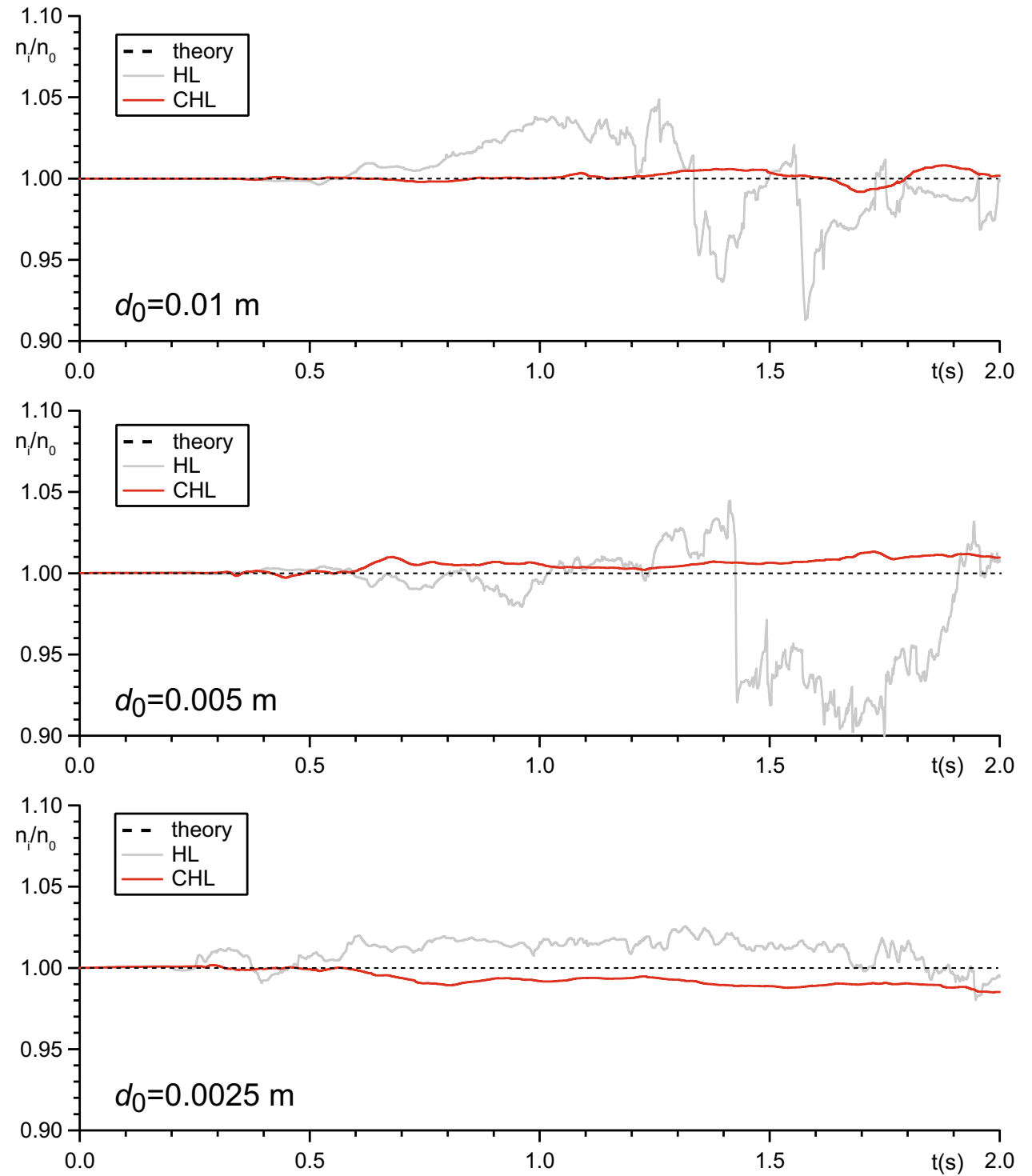

\subsection{Jet impingement on a flat plate (Molteni and Colagrossi 2009)}

The impingement of a water jet on a flat plate has been considered as a benchmark test to illustrate the enhanced pressure calculations by improved versions of both SPH (e.g., Molteni and Colagrossi 2009; Antuono et al. 2010) and MPS (e.g., Khayyer and Gotoh 2011) methods. A two-dimensional inviscid water jet impinges on a horizontal rigid plate. After the impact of jet and release of shock pressure, the flow regime becomes steady and the pressure at the stagnation point is that obtained from the Bernoulli equation.

Figure 8 shows a schematic sketch of calculation domain. A water jet of width $W=0.2 \mathrm{~m}$ impinges on a rigid flat plate from a distance of $D=2.5 \mathrm{~W}$ with a velocity of $U=$ $1.32 \mathrm{~m} / \mathrm{s}$. The particle size is considered to be $d_{0}=2.5 \mathrm{~mm}$.
The time increment of calculation, $\Delta t$, is set according to the Courant stability condition and a time resolution of $2.0 \mathrm{E}-4$.

Figure 9 shows the time histories of calculated pressure at measuring point $\mathrm{C}$ obtained by $\mathrm{HL}$ and $\mathrm{CHL}$ schemes. From this figure, the results by both HL and CHL appear to have the same level of accuracy. Thus, it may appear that the CHL scheme does not provide any enhancements for this benchmark test. However, some clear enhancements by CHL are observed when we consider the spatial distributions of particles and pressure field.

Figure 10 illustrates the spatial distributions of particles together with pressure field at $t=4.55 \mathrm{~s}$. From this figure, the instabilities (Fig. 10a1) and unphysical pressure oscillations (Fig. 10a2) seen in the vicinity of free surface corresponding to the HL scheme have been improved (Fig. 10b) by applying the CHL scheme. How- 
Fig. 5 Spatial distribution of particles together with pressure field for a $d_{0}=10 \mathrm{~mm}$,

b $d_{0}=5 \mathrm{~mm}$-designed

sinusoidal pressure

variations-irregular initial particle distribution
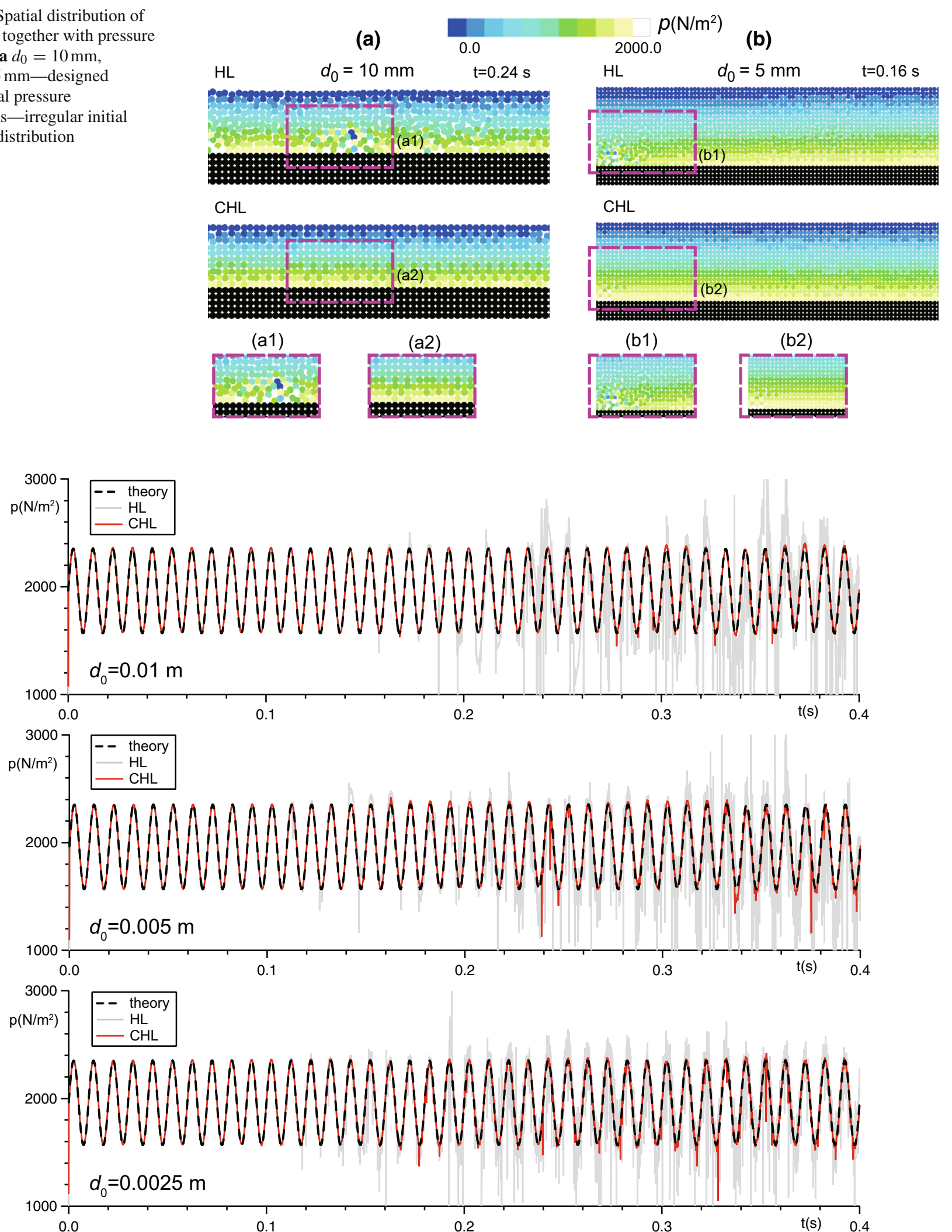

Fig. 6 Time histories of calculated pressure and analytical solution at measuring point A—designed sinusoidal pressure variations-irregular initial particle distribution 

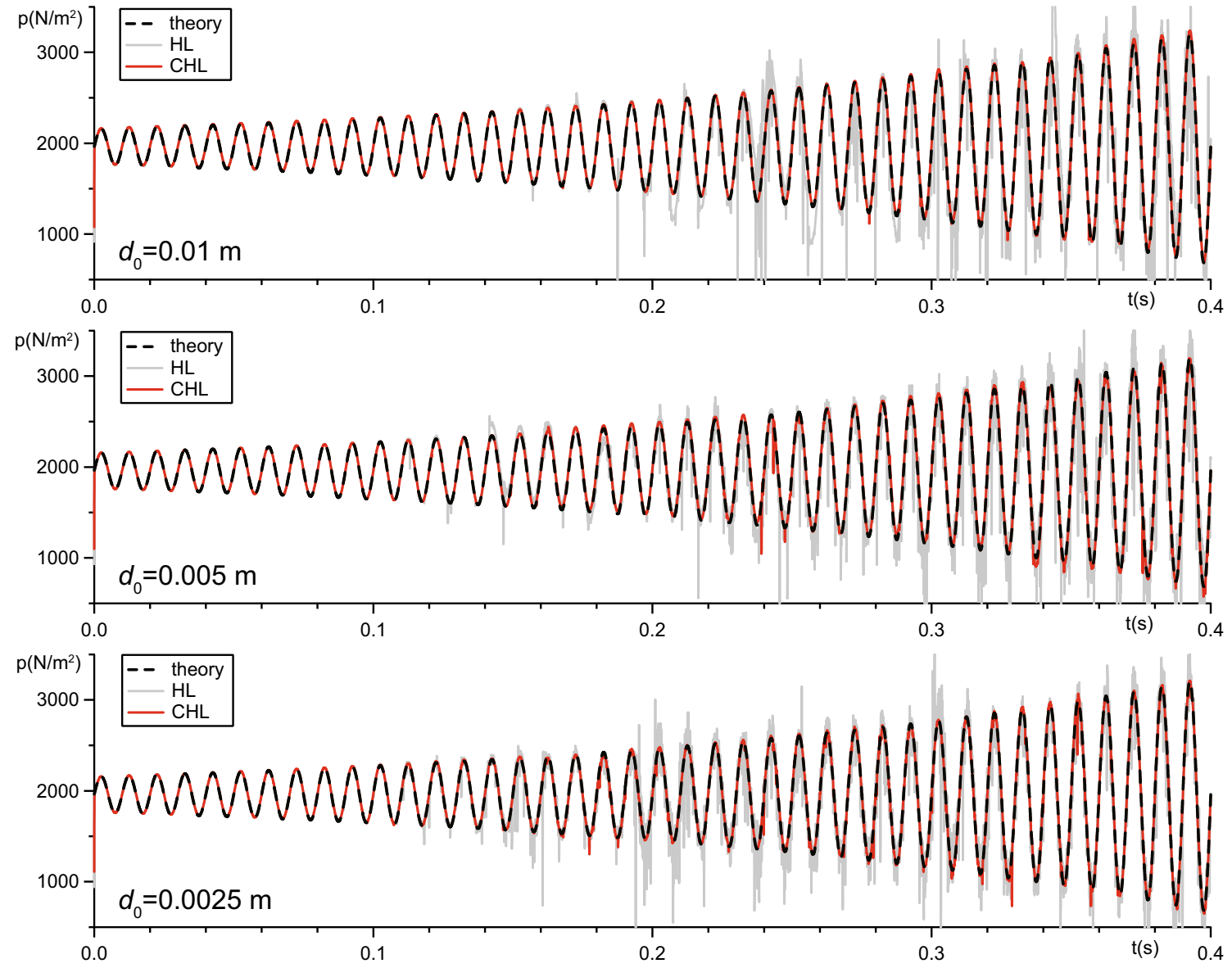

Fig. 7 Time histories of calculated and analytical pressure at measuring point A—designed exponentially excited sinusoidal pressure variations— irregular initial particle distribution

Table 1 RMSE (root mean square error) corresponding to $\mathrm{HL}$ and CHL

schemes-exponentially excited sinusoidal pressure variations

\begin{tabular}{|c|c|c|c|c|c|c|}
\hline \multirow{2}{*}{$\begin{array}{l}d_{0} \\
\text { Scheme }\end{array}$} & \multicolumn{2}{|l|}{$10 \mathrm{~mm}$} & \multicolumn{2}{|l|}{$5 \mathrm{~mm}$} & \multicolumn{2}{|l|}{$2.5 \mathrm{~mm}$} \\
\hline & HL & CHL & HL & CHL & HL & CHL \\
\hline Irregular & 234.5438 & 19.2850 & 162.1980 & 30.8089 & 150.9107 & 24.2292 \\
\hline
\end{tabular}

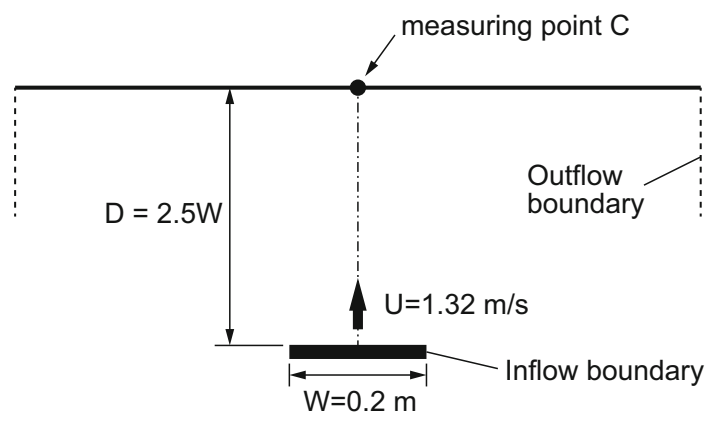

Fig. 8 Schematic sketch of the computational domain — a jet impinging on a flat plate

ever, consistent with the results in Fig. 9, both HL and CHL have resulted in almost similar spatial distributions of pressure at other regions. In the next section, the per-

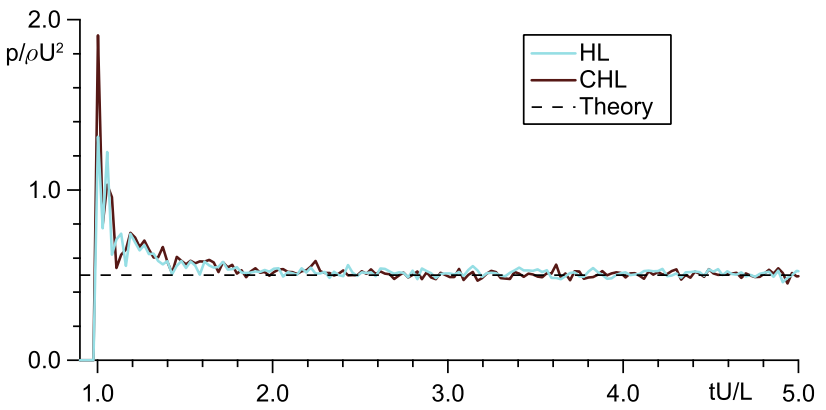

Fig. 9 Time histories of calculated pressure at measuring point $\mathrm{C}-\mathrm{a}$ jet impinging on a flat plate

formance of CHL with respect to HL is examined through the simulations of a deliberately perturbed jet impingement. 

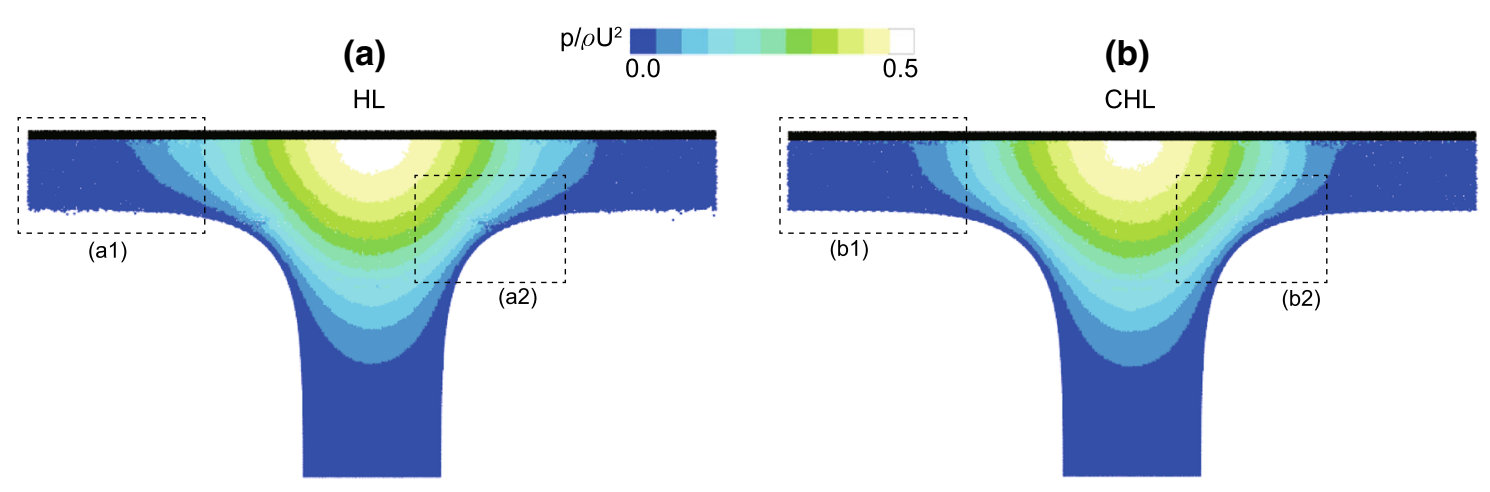

(a1)

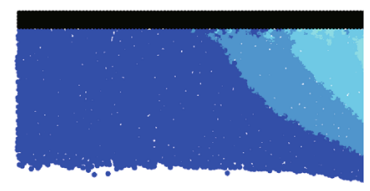

(b1)

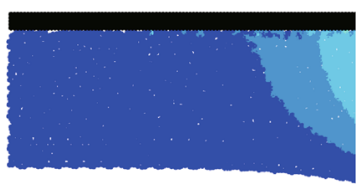

(a2)

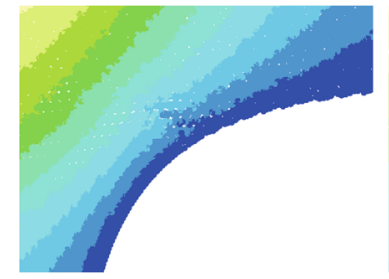

(b2)

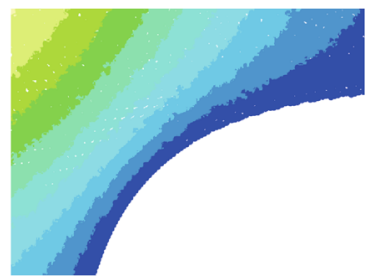

Fig. 10 Spatial distributions of particles together with pressure field at a typical time of $t=4.55 \mathrm{~s}-\mathrm{a}$ jet impinging on a flat plate

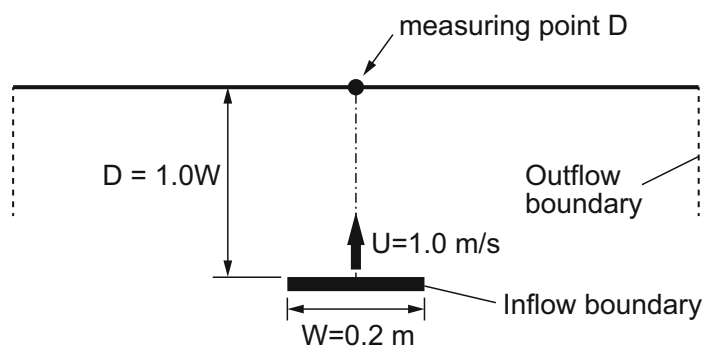

Fig. 11 Schematic sketch of the computational domain —a perturbed jet impinging on a flat plate

\subsection{Perturbed jet impingement on a flat plate}

A deliberately perturbed jet impinging on a flat plate is reproduced to demonstrate the enhanced performance of CHL with respect to HL. A schematic sketch of calculation domain is shown in Fig. 11. A perturbed water jet of width $W=0.2 \mathrm{~m}$ impinges on a rigid flat plate from a distance of $D=1.0 \mathrm{~W}$ with a velocity of $U=1.0 \mathrm{~m} / \mathrm{s}$. A set of spatial resolutions of $d_{0}=1.25,2.0$ and $2.5 \mathrm{~mm}$ is considered. The perturbation in the water jet is caused by deliberately assigning an irregular distribution to the initial 5 layers of inflow boundary particles by randomly displacing them by $0.2 d_{0}$ in vertical direction. This irregular initial distribution is exactly the same for both HL and CHL simulations. The calculation time step for all the performed simulations of this section is chosen by considering the Courant stability condition and a maximum allowable time resolution of $2.0 \mathrm{E}-4$.

Figure 12 shows the snapshots of particles together with pressure field at $t=0.12$ and 0.20 s by HL and CHL schemes.
As it can be seen from the presented figure, just after the impact instant at $t=0.20 \mathrm{~s}$, the pressure field by the HL scheme is characterized by clear discrepancies and spuriously calculated pressures. Such discrepancies are clearly minimized in the snapshot by the CHL scheme.

Figure 13 shows the snapshots of particles and pressure fields at $t=0.20 \mathrm{~s}$ by the HL and CHL schemes corresponding to two other considered spatial resolutions. In both cases, the enhanced performance of CHL with respect to $\mathrm{HL}$ is evident.

Figure 14 depicts the time history of calculated pressures at measuring point $\mathrm{D}$ by the $\mathrm{HL}$ and $\mathrm{CHL}$ schemes corresponding to $d_{0}=2.0 \mathrm{~mm}$. Although results of a perturbed jet impact tend to contain more numerical noises in comparison with an unperturbed, perfectly smooth jet (with a completely uniform velocity field), the CHL is shown to outperform the $\mathrm{HL}$ in a quantitative comparison as well.

The superiority of CHL with respect to HL in simulation of a perturbed jet impingement suggests that this scheme should be preferred to the HL scheme in simulations of violent fluid flows that often contain irregularities and fragmentations.

\subsection{Two-dimensional diffusion problem}

In order to further verify the accuracy of both HL and CHL schemes, a two-dimensional diffusion problem corresponding to a square domain subjected to Dirichlet boundary condition is simulated. Figure 15 shows a schematic sketch of this test. The Dirichlet boundary condition, specified by $\phi=z$ at the square's four sides, is set as $z=0$ except for the horizontal side at $y=0$, where the condition of $z=x$ 

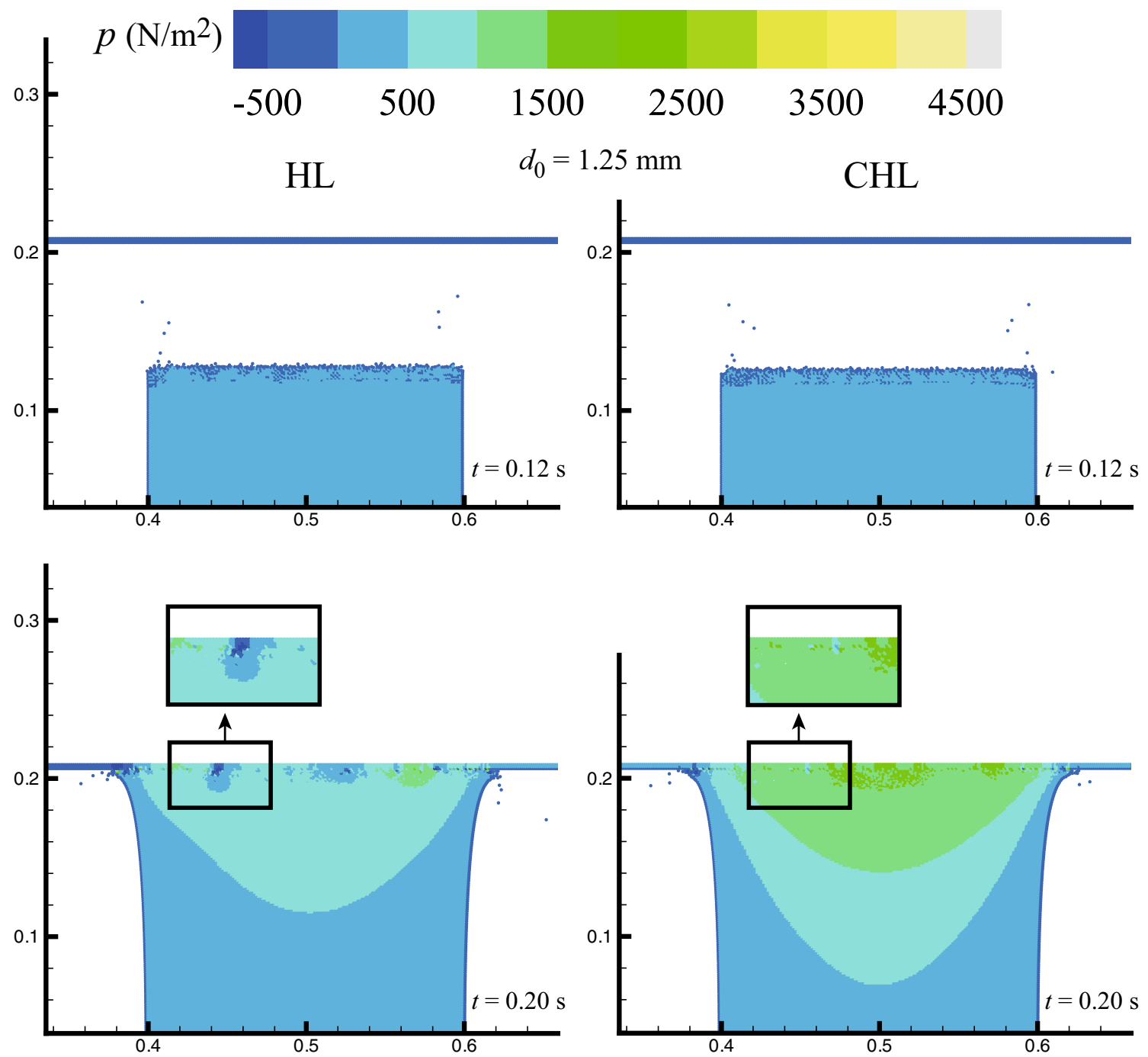

Fig. 12 Spatial distributions of particles together with pressure field at $t=0.12$ and $0.2 \mathrm{~s}$ corresponding to $d_{0}=1.25 \mathrm{~mm}-\mathrm{a}$ perturbed jet impinging on a flat plate

is imposed. The initial condition for all the other particles is set as $\phi=0$. The exact solution for this diffusion problem is given as (Young et al. 2005):

$$
\begin{aligned}
\phi(x, y)= & \sum_{n=1}^{\infty}\left(\frac{2(-1)^{n+1}}{n \pi \sinh (n \pi)}\right. \\
& \times \sinh \{n \pi(1-y)\} \sinh (n \pi x))
\end{aligned}
$$

Two different spatial resolutions are considered, namely, $L / D=10$ and $L / D=100$ with $L$ being the square's side length (taken as unity) and $D$ being the particle diameter $\left(d_{0}\right)$, respectively. Figure 16 shows the spatial distributions of calculated $\phi$ corresponding to these two resolutions by applying the CHL scheme. For this test, the HL scheme resulted in almost the same qualitative results.

Figure 17 shows a quantitative comparison in between the HL and CHL schemes with respect to analytical solu- tion for $L / D=10$ at $x=0.5$ and at $y=0.5$. From the presented figure, the CHL scheme has provided relatively more accurate results for this considered resolution.

Figure 18 shows the calculation results for the fine resolution case of $L / D=100$. For this calculation case, both HL and CHL schemes have provided almost accurate results. In order to quantify the accuracy, the RMSE of calculated results with respect to the analytical solution is presented in Table 2. In both cases, the CHL scheme shows a smaller RMSE compared with the HL scheme.

\section{Concluding remarks}

The paper presents a corrected higher order Laplacian (CHL) model for enhancement of pressure calculation by moving particle semi-implicit (MPS) method. The proposed CHL 
Fig. 13 Spatial distributions of particles together with pressure field at $t=0.2 \mathrm{~s}$ corresponding to $d_{0}=2.0 \mathrm{~mm}$ and $2.5 \mathrm{~mm}-\mathrm{a}$ perturbed jet impinging on a flat plate

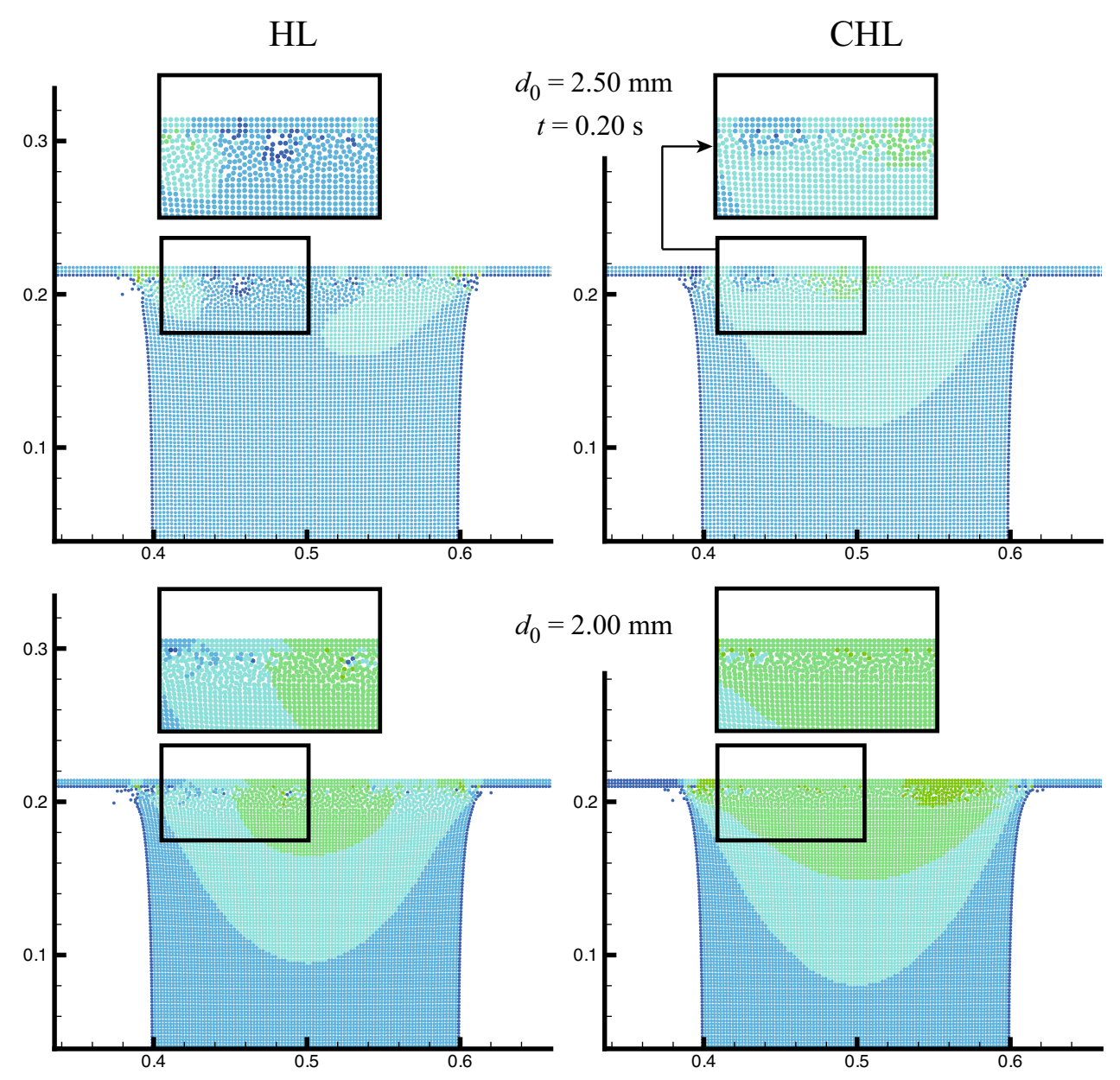

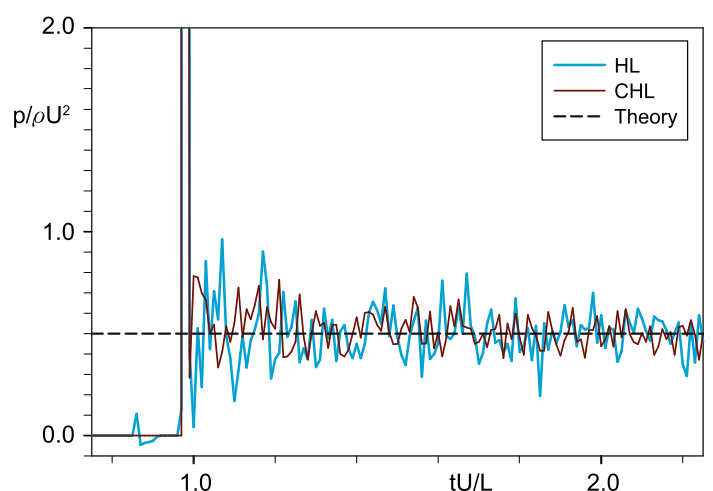

Fig. 14 Time histories of calculated pressure at measuring point $\mathrm{D}-\mathrm{a}$ perturbed jet impinging on a flat plate

scheme is derived by meticulously taking the divergence of a corrected SPH gradient model in a similar manner to derivation of higher order Laplacian (HL) scheme conducted by Khayyer and Gotoh $(2010,2012)$. The considered corrected SPH gradient is characterized by a corrective matrix to assure the first-order consistency of pressure gradient approximations.

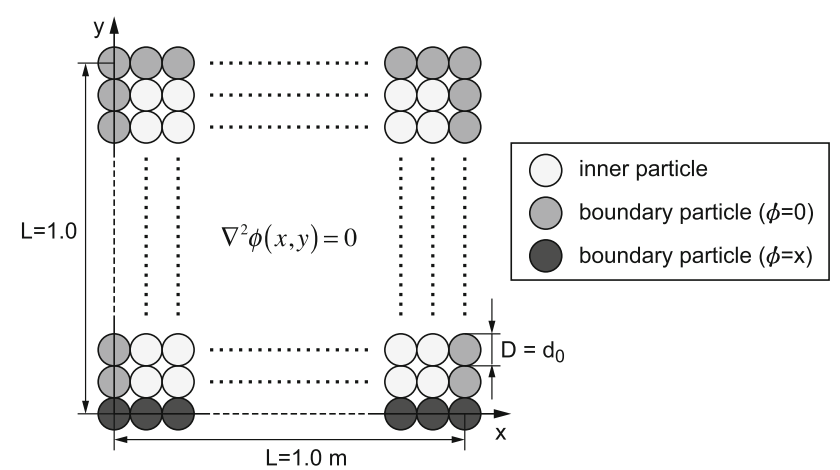

Fig. 15 Schematic sketch of calculation domain-a 2D diffusion problem

The enhanced performance of CHL with respect to HL is shown by performing designed sinusoidal and exponentially excited sinusoidal pressure variations (Khayyer and Gotoh 2012), unperturbed/perturbed jets impinging on a flat plate (Molteni and Colagrossi 2009) and a 2D diffusion problem (Young et al. 2005) through both qualitative and quantitative comparisons. The superiority of $\mathrm{CHL}$ with respect to $\mathrm{HL}$ is found to be more realizable in presence of irregu- 
larities in particle distributions or highly accelerated flow fields, often encountered in simulation of violent fluid flows. Thus, despite relative complexity in formulation and coding, the CHL scheme should be preferred to the HL one, in simulations related to practical engineering applications,

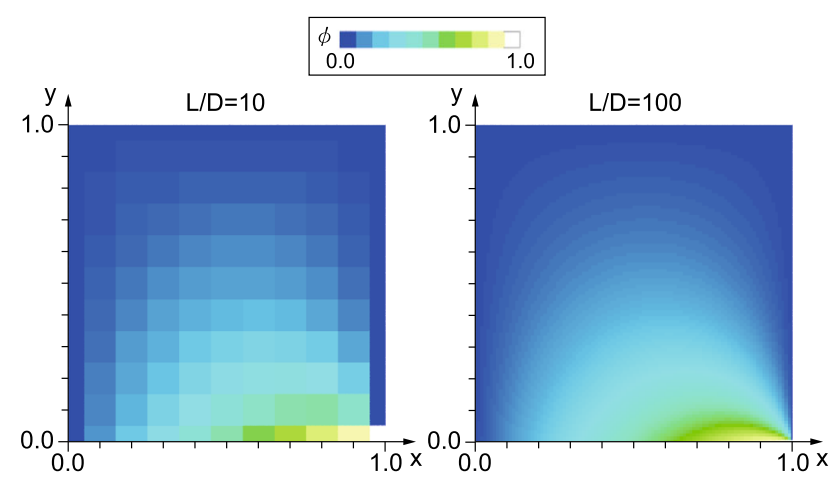

Fig. 16 Spatial distributions of calculated $\phi$ by the CHL scheme-a 2D diffusion problem including those related to ocean engineering. More rigorous studies on the accuracy and convergence of both HL and CHL schemes are scheduled to be conducted by the authors.

Although in this paper, derivation and application of CHL is considered for the MPS method, similar developments can be easily made for another well-known projection-based particle method, i.e., incompressible SPH method (e.g., Shao and Lo 2003).

Development of refined differential operator models, such as CHL, will be also helpful to achieve a more accurate and more reliable reproduction of SPS (subparticle scale) turbulence (Gotoh et al. 2001) in hydrodynamic fluid flows where presence of unphysical pressure oscillations remains to be a challenging difficulty (Gotoh and Sakai 2006). Upon achieving an accurate and fully reliable MPS-based solver, real-time fluid flow simulations are expected to be obtained via high-performance GPU (graphics processing unit)-based computations (e.g.,
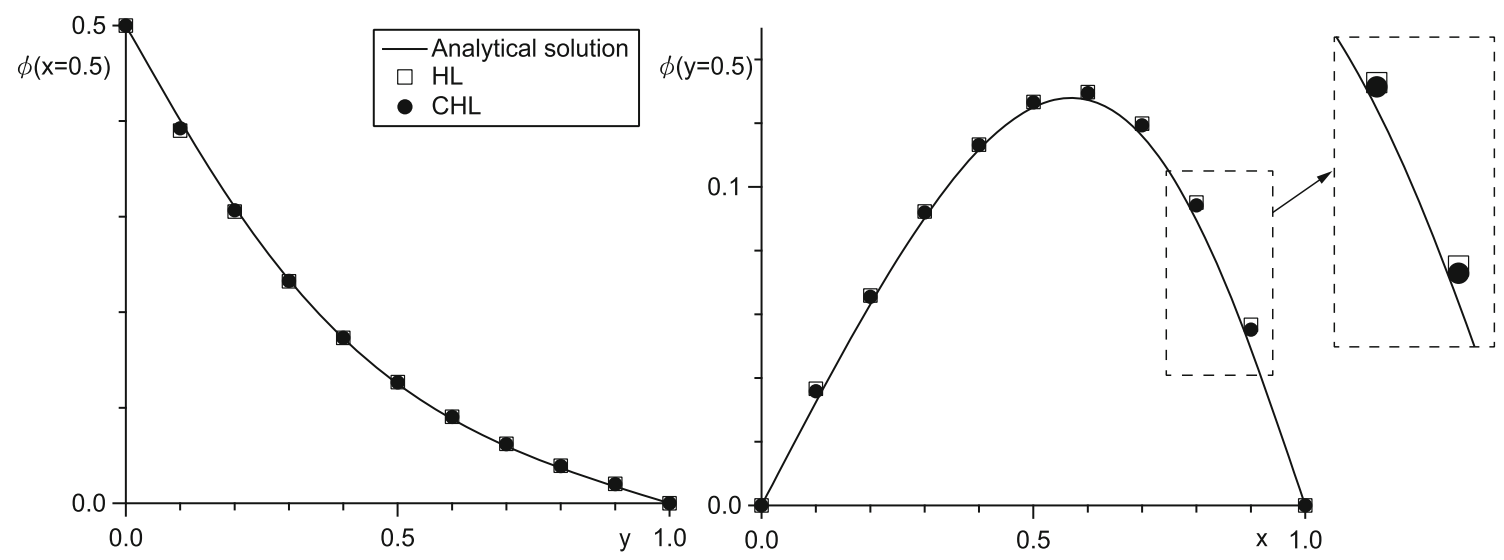

Fig. 17 Quantitative comparison in between the HL and CHL schemes with respect to analytical solution for $L / D=10$ at $x=0.5$ and at $y=0.5-\mathrm{a} 2 \mathrm{D}$ diffusion problem
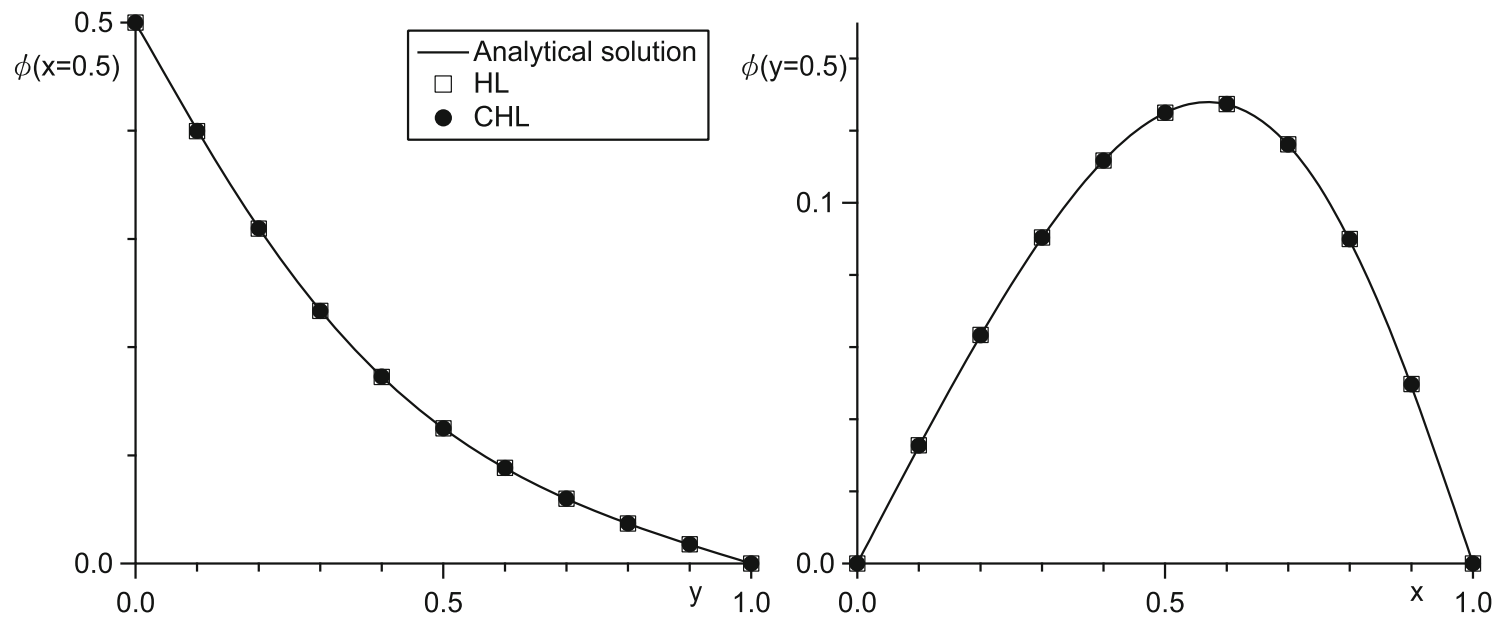

Fig. 18 Calculation results by the HL and CHL schemes for $L / D=100$ at $x=0.5$ and at $y=0.5-\mathrm{a} 2 \mathrm{D}$ diffusion problem 
Table 2 RMSE (root mean square error) corresponding to HL and CHL schemes-a 2D diffusion problem

\begin{tabular}{lllll}
\hline$L / D=10$ & & & $L / D=100$ & \\
\cline { 1 - 2 } & CHL & & HL & CHL \\
\hline 0.00748421 & 0.00649334 & & 0.00125827 & 0.00105165 \\
\hline
\end{tabular}

Hori et al. 2011). Gotoh (2009) and Koshizuka (2011) provide comprehensive reviews on key issues for extension of particle methods for practical engineering applications.

\section{References}

Adami S, Hu XY, Adams NA (2012) A generalized wall boundary condition for smoothed particle hydrodynamics. J Comput Phys 231(21):7057-7075

Antuono M, Colagrossi A, Marrone S, Molteni D (2010) Free-surface flows solved by means of SPH schemes with numerical diffusive terms. Comput Phys Commun 181(3):532-549

Antuono M, Colagrossi A, Marrone S (2012) Numerical diffusive terms in weakly-compressible SPH schemes. Comput Phys Commun 183(12):2570-2580

Antuono M, Bouscasse B, Colagrossi A, Marrone S (2014) A measure of spatial disorder in particle methods. Comput Phys Commun 185(10):2609-2621

Bonet J, Lok TS (1999) Variational and momentum preservation aspects of smooth particle hydrodynamic formulation. Comput Methods Appl Mech Eng 180:97-115

Chen JK, Beraun JE, Jih CJ (1999) An improvement for tensile instability in smoothed particle hydrodynamics. Comput Mech 23:279-287

Colagrossi A, Landrini M (2003) Numerical simulation of interfacial flows by smoothed particle hydrodynamics. J Comput Phys 191(2):448-475

Delorme L, Colagrossi A, Souto-Iglesias A, Zamora-Rodriguez R, Botia-Vera E (2009) A set of canonical problems in sloshing, part I: pressure field in forced roll-comparison between experimental results and SPH. Ocean Eng 36(2):168-178

Gingold RA, Monaghan JJ (1977) Smoothed particle hydrodynamics: theory and application to non-spherical stars. Mon Not R Astron Soc 181:375-89

Gotoh H, Shibahara T, Sakai T (2001) Sub-particle-scale turbulence model for the mps method-Lagrangian flow model for hydraulic engineering. Comput Fluid Dyn J 9(4):339-347

Gotoh H, Sakai T (2006) Key issues in the particle method for computation of wave breaking. Coast Eng 53:171-179

Gotoh H (2009) Lagrangian particle method as advanced technology for numerical wave flume. Int J Offshore Polar Eng 19(3):161-167

Gotoh H, Khayyer A, Ikari H, Arikawa T, Shimosako K (2014) On enhancement of Incompressible SPH method for simulation of violent sloshing flows. Appl Ocean Res 46:104-115

Gotoh H, Okayasu A, Watanabe Y (2013) Computational wave dynamics. World Scientific Publishing Co, Singapore

Hori C, Gotoh H, Ikari H, Khayyer A (2011) GPU-acceleration for moving particle semi-implicit method. Comput Fluids 51(1):174183

Hu XY, Adams NA (2009) A constant-density approach for incompressible multi-phase SPH. J Comput Phys 228(6):2082-2091
Hwang SC, Khayyer A, Gotoh H, Park JC (2014) Development of a fully Lagrangian MPS-based coupled method for simulation of fluid-structure interaction problems. J Fluids Struct 50:497-511

Khayyer A, Gotoh H, Shao SD (2008) Corrected Incompressible SPH method for accurate water-surface tracking in breaking waves. Coast Eng 55(3):236-250

Khayyer A, Gotoh H (2009a) Modified moving particle semi-implicit methods for the prediction of 2D wave impact pressure. Coast Eng $56: 419-440$

Khayyer A, Gotoh H (2009b) Wave impact pressure calculations by improved SPH methods. Int J Offshore Polar Eng 19(4):300-307

Khayyer A, Gotoh H (2010) A higher order Laplacian model for enhancement and stabilization of pressure calculation by the MPS method. Appl Ocean Res 32(1):124-131

Khayyer A, Gotoh H (2011) Enhancement of stability and accuracy of the moving particle semi-implicit method. J Comput Phys 230:3093-3118

Khayyer A, Gotoh H (2012) A 3D higher order Laplacian model for enhancement and stabilization of pressure calculation in 3D MPSbased simulations. Appl Ocean Res 37:120-126

Khayyer A, Gotoh H (2013) Enhancement of performance and stability of MPS meshfree particle method for multiphase flows characterized by high density ratios. J Comput Phys 242:211-233

Kondo M, Koshizuka S (2011) Improvement of stability in moving particle semi-implicit method. Int J Numer Methods Fluids 65: 638-654

Koshizuka S, Oka Y (1996) Moving particle semi-implicit method for fragmentation of incompressible fluid. Nucl Sci Eng 123:421434

Koshizuka S (2011) Current achievements and future perspectives on particle simulation technologies for fluid dynamics and heat transfer. J Nucl Sci Technol 48(2):155-168

Lind S, Xu R, Stansby P, Rogers B (2012) Incompressible smoothed particle hydrodynamics for free-surface flows: a generalised diffusion-based algorithm for stability and validations for impulsive flows and propagating waves. J Comput Phys 231(4):14991523

Liu WK, Adee J, Jun S (1993) Reproducing kernel and wavelets particle methods for elastic and plastic problems. Adv Comput Methods Mater Model 180(268):175-190

Molteni D, Colagrossi A (2009) A simple procedure to improve the pressure evaluation in hydrodynamic context using the SPH. Comput Phys Commun 180(6):861-872

Monaghan JJ (1992) Smoothed particle hydrodynamics. Ann Rev Astron Astrophys 30:543-574

Oger G, Doring M, Alessandrini B, Ferrant P (2007) An improved SPH method: towards higher order convergence. J Comput Phys 225(2):1472-1492

Randles PW, Libersky LD (1996) Smoothed particle hydrodynamics: some recent improvements and applications. Comput Meth Appl Mech Eng 139:375-408

Shao SD, Lo EYM (2003) Incompressible SPH method for simulating Newtonian and non-Newtonian flows with a free surface. Adv Water Resour 26(7):787-800

Souto-Iglesias A, Macià F, González LM, Cercos-Pita JL (2013) On the consistency of MPS. Comput Phys Commun 184(3):732-745

Touzé DL, Colagrossi A, Colicchio G, Greco M (2013) A critical investigation of smoothed particle hydrodynamics applied to problems with free surfaces. Int J Numer Methods Fluids 73:660-691

Tsuruta N, Khayyer A, Gotoh H (2013) A short note on dynamic stabilization of moving particle semi-implicit method. Comput Fluids $82: 158-164$

Tsuruta N, Khayyer A, Gotoh H (2015) Space potential particles to enhance the stability of projection-based particle methods. Int J Comput Fluid Dyn. doi:10.1080/10618562.2015.1006130 
Ulrich C, Leonardi M, Rung T (2013) Multi-physics SPH simulation of complex marine-engineering hydrodynamic problems. Ocean Eng 64:109-121

Veen D, Gourlay T (2012) A combined strip theory and smoothed particle hydrodynamics approach for estimating slamming loads on a ship in head seas. Ocean Eng 43:64-71
Wendland H (1995) Piecewise polynomial, positive definite and compactly supported radial functions of minimal degree. Adv Comput Math 4:389-396

Young DL, Chen KH, Lee CW (2005) Novel meshless method for solving the potential problems with arbitrary domain. J Comput Phys 209:290-321 